\title{
Analysis of Risk Factors Related to Acute Subarachnoid Hemorrhage After Spinal Surgery
}

Jianquan Zhao

Shanghai Changzheng Hospital

Heng Jiang

Shanghai Changzheng Hospital

Yichen Meng

Shanghai Changzheng Hospital

Rui Gao

Shanghai Changzheng Hospital

Jun Ma

Shanghai Changzheng Hospital

Ce Wang

Shanghai Changzheng Hospital

Xuhui Zhou ( $\sim$ spine415@163.com)

Shanghai Changzheng Hospital https://orcid.org/0000-0003-2847-952X

\section{Research Article}

Keywords: subarachnoid hemorrhage (SAH), cerebrospinal fluid(CSF) , hypertension, drainage

Posted Date: August 23rd, 2021

DOl: https://doi.org/10.21203/rs.3.rs-800833/v1

License: (c) (i) This work is licensed under a Creative Commons Attribution 4.0 International License.

Read Full License

Version of Record: A version of this preprint was published at World Neurosurgery on December 1st, 2021. See the published version at https://doi.org/10.1016/j.wneu.2021.12.098. 


\section{Abstract}

Study Design: A retrospective single-center study.

Background: Subarachnoid hemorrhage (SAH) is a rare complication secondary to cerebrospinal fluid (CSF) leakage during spinal surgery, but the specific cause of the bleeding is still unclear.

Objective冈ln this study, we studied cases of acute SAH after spinal surgery to find the related risk factors for bleeding.

Methods: A total of 441 patients with CSF leakage who underwent spinal surgery in [BLINDED FOR REVIEW] from 2011 to 2020 were retrospectively analyzed. According to whether postoperative SAH occurred, they were divided into SAH group and Control group. By comparing the two groups of demographic data, past history, imaging data, intraoperative findings, perioperative complications, treatment conditions to find the risk factors of SAH, to provide guidance for the prevention of SAH after spinal surgery.

Results: In SAH group, 17 cases (73.9\%) had preoperative hypertension and 3 cases $(13.0 \%)$ had diabetes. The intraoperative CSF leakage was about $118.4 \pm 56.9 \mathrm{ml}$. The mean postoperative drainage was $15.4 \pm 5.8 \mathrm{ml} / \mathrm{h}$; Compared with SAH group, Control group had 123 cases (29.4\%) with hypertension before operation, 40 cases $(9.6 \%)$ with diabetes, intraoperative CSF leakage was about $76.3 \pm 23.0 \mathrm{ml}$, and the mean postoperative continuous drainage $9.7 \pm 2.1 \mathrm{ml} / \mathrm{h}$. Binary logistic regression analysis showed that hypertension, intraoperative CSF leakage, and postoperative CSF continuous drainage speed are related to $\mathrm{SAH}$.

Conclusion: The rapid leakage of CSF caused by the rupture of the dural sac during operation and the rapid drainage of CSF after surgery are closely related to the occurrence of such complications. In addition, hypertension is a related factor of SAH during spinal surgery.

\section{Introduction:}

Subarachnoid hemorrhage (SAH) is a clinical syndrome caused by the rupture of the diseased blood vessels in the brain base or brain surface, and the blood flow directly into the subarachnoid space ${ }^{1}$. SAH can have fatal consequences if it is not recognized and treated early. SAH after spinal surgery is rare, and the symptoms at the beginning of the onset are atypical and can only be detected by accidental CT or MRI. Therefore, early detection and treatment are difficult.

The cause of SAH is mainly aneurysms, accounting for about $85 \%$ of all cases. Other causes include nonaneurysmal hemorrhage around the midbrain, vascular malformation, dural arteriovenous fistula, coagulopathy, and smoking cocaine and pituitary apoplexy ${ }^{2-3}$. However, the medical records of SAH after spinal surgery reported in the previous literature are less than 100 cases, and most of them are case reports, which cannot provide sufficient guidance for the etiology and prognosis of the disease. 
At present, there is no studies on risk factors related to SAH after spinal surgery in the world. This article retrospectively analyzed the patients who underwent spinal surgery from 2011 to 2020 in [BLINDED FOR REVIEW], and analyzed the risk factors of SAH in patients with CSF leakage through the largest casecontrol study. Our research will help spine surgeons better understand the relationship between spinal surgery and SAH, and provide guidance for the prevention of SAH after spinal surgery.

\section{Methods:}

This study was approved by the IRB of [BLINDED FOR REVIEW], and all patients gave valid consent to participate. 14526 consecutive patients who underwent spine surgery from January 2011 to December 2020 in [BLINDED FOR REVIEW] were enrolled in this retrospective cohort study. Among them, 441 patients had intraoperative cerebrospinal fluid leakage. Patients with previous history of cerebral hemorrhage, aneurysmal, and cerebral infarction were excluded from this study. According to whether postoperative SAH occurred, it was divided into two groups: 23 patients with subarachnoid hemorrhage (SAH group), and 418 patients without subarachnoid hemorrhage served as control group.

When cerebrospinal fluid leakage was found intraoperatively, the drainage bottle was replaced immediately for separate intraoperative measurement of cerebrospinal fluid. The incision subfascial drainage tube was routinely placed for incision drainage after surgery. Postoperatively, All patients were treated with positive pressure drainage by metering drainage bag, cerebrospinal fluid drainage is generally not restricted. Cervical spine surgery patients were required to maintain recumbent position without pillow, and thoracic and lumbar spine surgery patients were in trendelenburg position. Patients with severe headache, nausea, vomiting, changes of consciousness and positive meningeal stimulation signs after surgery were immediately given head CT to determine whether there was subarachnoid hemorrhage, if not, head MRI examination would be performed, and some patients received DSA examination.

Record the clinical data, intraoperative findings and disease prognosis of all patients, including: general population information, past history, operation time, blood loss, type of spinal surgery, whether there is dural rupture during the operation, the location of the rupture, treatment strategies, and drainage Tube placement, postoperative drainage, neurological symptoms, symptom treatment strategies and prognosis.

\section{Statistical Analysis:}

Descriptive data analysis was conducted depending on the nature of the considered criteria. For quantitative data, this included the number of observed values (and missing, if any), mean, standard deviation, median, first and third quartiles, and minimum and maximum. For qualitative data, this included the number of observed values (and missing, if any) and the number and percentage of patients per class. The characteristics of the studied population were described according to the presence or lack of SAH. A binary logistic regression was performed to identify risk factors associated with SAH. Factors 
initially considered were those selected as statistically significant at the $25 \%$ threshold in a first univariate approach or those previously described in the literature as an important risk factor. A stepwise descending strategy was then applied from the initial full model to determine the most parsimonious one, removing step-by-step all the nonstatistically significant parameters and keeping the clinically relevant parameters and the confounding factors. All statistical analyses were performed using SPSS statistical software v. 25.0 (IBM, Armonk, New York, USA) with the level of statistical significance set at $P<0.05$.

\section{Results:}

The mean age of patients in the SAH group was $52.9 \pm 15.3$ years. There were 17 cases of preoperative hypertension and 3 cases of diabetes. The preoperative BMI was $22.1 \pm 5.2$. The mean age of the control group was $51.2 \pm 17.2$ years old. There were 123 cases of hypertension and 40 cases of diabetes before the operation. The BMI before the operation was $20.9 \pm 6.1$.

In the SAH group, there was 1 case of cervical spondylosis complicated with dural sac ossification. The dural defect was large without suture after anterior resection of the ossified tissue. The remaining 22 cases were repaired by the dural sac. The CSF leakage during the operation was about $118.4 \pm 56.9 \mathrm{ml}$. Drainage tubes were placed for continuous drainage after the operation. The mean hourly CSF leakage before neurological symptoms was $15.4 \pm 5.8 \mathrm{ml} / \mathrm{hr}$. In control group, 22 cases did not suture the dural sac, and the remaining 396 cases underwent intraoperative dural sac repair. The intraoperative CSF leakage was about $76.3 \pm 23.0 \mathrm{ml}$. After the operation, the mean CSF leakage was $9.7 \pm 2.1 \mathrm{ml} / \mathrm{hr}$. Preoperative and intraoperative individual characteristics of patients, is presented in Tables $1 \& 2$. 
Table 1

Preoperative Individual Characteristics of

Patients With and Without SAH

\begin{tabular}{|lcl|}
\hline & \multicolumn{2}{|c|}{ SAH } \\
\cline { 2 - 3 } & YES(n= 23) & NO(n= 418) \\
\hline Sex & & \\
\hline Male & $14(60.9 \%)$ & $244(58.4 \%)$ \\
\hline Female & $9(39.1 \%)$ & $174(41.6 \%)$ \\
\hline Age & $52.9 \pm 15.3$ & $51.2 \pm 17.2$ \\
\hline$<50$ & $7(30.4 \%)$ & $104(24.9 \%)$ \\
\hline$\geq 50$ & $16(69.6 \%)$ & $314(75.1 \%)$ \\
\hline BMI & $22.1 \pm 5.2$ & $20.9 \pm 6.1$ \\
\hline$<25$ & $11(47.8 \%)$ & $198(47.4 \%)$ \\
\hline$\geq 25$ & $12(52.3 \%)$ & $220(52.3 \%)$ \\
\hline Hypertension & \\
\hline Yes & $17(73.9 \%)$ & $123(29.4 \%)$ \\
\hline No & $6(26.1 \%)$ & $295(70.6 \%)$ \\
\hline Diabetes & & \\
\hline Yes & $3(13.0 \%)$ & $40(9.6 \%)$ \\
\hline No & $20(87.0 \%)$ & $378(90.4 \%)$ \\
\hline a Data are presented as $\mathrm{n}(\%)$ or mean \pm SD. \\
\hline
\end{tabular}


Table 2

Intraoperative Characteristics of Patients With and Without SAH

\begin{tabular}{|lll|}
\hline & SAH \\
\cline { 2 - 3 } & YES( $\mathbf{n = 2 3 )}$ & NO( $\mathbf{n = 4 1 8})$ \\
\hline Intraoperative CSF leakage $(\mathrm{ml})$ & $118.4 \pm 56.9$ & $76.3 \pm 23.0$ \\
\hline Intraoperative repair & & \\
Yes & $22(95.7 \%)$ & $396(94.7 \%)$ \\
\hline No & $1(4.3 \%)$ & $22(5.3 \%)$ \\
\hline Postoperative CSF drainage speed (ml/hour) & $15.4 \pm 5.8$ & $9.7 \pm 2.1$ \\
\hline Operation time(hours) & $3.5 \pm 0.8$ & $3.3 \pm 0.7$ \\
\hline Intraoperative blood loss $(\mathrm{ml})$ & $251 \pm 59.9$ & $231.7 \pm 74.7$ \\
\hline a Data are presented as $\mathrm{n}(\%)$ or mean \pm SD. & & \\
\hline
\end{tabular}

Through univariate analysis, the following factors reached the $25 \%$ correlation threshold and were included in the binary logistic regression analysis: hypertension, CSF leakage, postoperative CSF drainage speed, and operation time. In the binary logistic regression analysis, hypertension, CSF leakage and postoperative CSF drainage speed were correlated with the increase of SAH $(P \leq 0.05)$ (Table 3).

Table 3

Binary logistic regression analysis of risk factors

\begin{tabular}{|lllll|}
\hline & Comparison & Odds Ratio & $95 \%$ Cl & P Value \\
\hline Hypertension & Yes vs no & 3.157 & $1.125-6.978$ & 0.047 \\
\hline Intraoperative CSF leakage & $<100 \mathrm{ml} \mathrm{vs} \geq 100 \mathrm{ml}$ & 1.036 & $1.012-1.060$ & 0.004 \\
\hline Postoperative CSF drainage speed & $<10 \mathrm{ml} / \mathrm{h}$ vs $\geq 100 \mathrm{ml}$ & 1.400 & $1.063-1.845$ & 0.017 \\
\hline Operation time & $<3 \mathrm{~h}$ vs $\geq 3 \mathrm{~h}$ & 3.178 & $0.889-11.361$ & 0.075 \\
\hline a Data are presented as n (\%) or mean \pm SD. & & & \\
\hline
\end{tabular}

All 23 patients in the SAH group developed neurological symptoms after surgery. Symptoms included 19 cases of headache, 14 cases of vomiting, 1 case of aphasia, and 13 cases of altered consciousness. In one case, the intraoperative drainage volume was $231 \mathrm{ml}$, and the anesthesia resuscitation time was longer. The emergency CT scan of the head showed intraoperative SAH. The remaining patients developed symptoms on the first 2-3 days after surgery. All patients had stable hemodynamics during and after operation, and no hypertension or hypotension. After the symptoms appeared, CT scan showed SAH, including 3 cases with subdural hemorrhage. In control group, 292 patients had neurological symptoms after operation, including 244 cases of headache, 121 cases of vomiting, 9 cases of changes in consciousness, and 5 cases of postoperative cerebral infarction. 
The 16 patients in the SAH group recovered completely without residual neurological dysfunction. 6 patients had partial neurological dysfunction. One patient found a SAH on the first day after surgery, and a subdural hematoma on the third day after surgery. The patient was declared brain dead on the 20th day after surgery. Five patients in control group had postoperative cerebral infarction, of which three died.

\section{Discussion:}

This study is the largest number of cases at present. The main finding is that the incidence of SAH after spinal surgery is $0.15 \%(23 / 14526)$ in patients with spinal surgery and $5.2 \%(23 / 441)$ in patients with CSF leakage. Another important finding is that hypertension, intraoperative CSF leakage and postoperative CSF drainage time are related to the occurrence of SAH after spinal surgery.

At present, the pathophysiological mechanism of postoperative SAH is not clear. Previous studies have reported that hypertension is a risk factor for SAH. Long-term hypertension leads to rupture of deep penetrating vessels, and common sites include basal ganglia, thalamus, brainstem and cerebellum². Bozkurt suggested that elderly patients with hypertension and brain atrophy were more prone to $\mathrm{SAH}^{4}$. Canao believed that the risk of non-aneurysmal SAH in hypertensive patients was 2.6 times higher than that in control group ${ }^{5}$. Their report was also confirmed in our study, $73.9 \%$ of patients in the SAH group suffered from hypertension before surgery. Binary Logistic regression analysis suggested that cerebrovascular lesions caused by hypertension were the risk factors for SAH after spinal surgery.

Studies have speculated that the cause of SAH after spinal surgery is related to excessive CSF drainage ${ }^{6}$. A large amount of CSF loss will lead to brain dehydration, further stretching of the bridging vein, and finally rupture of the bridging vein and bleeding. Lower intracranial pressure due to CSF leakage can also cause intracranial hemorrhage, including $\mathrm{SAH}$, subdural hemorrhage, parenchymal hemorrhage, and intraventricular hemorrhage. This phenomenon is most likely the result of a combination of factors, with a decrease in CSF volume causing the brain and spine to sag, thereby tensing the superior cerebellar and bridge veins. At the same time, the compensatory increase in venous volume leads to vascular filling and further expansion of venous structure, which is prone to venous avulsion or other damage ${ }^{7-8}$. Therefore, many scholars believe that intraoperative CSF leakage is associated with postoperative SAH. However, it is not fully understood which factors are involved in the specific mechanism of SAH after intraoperative CSF leakage. In our case study, from the perspective of the amount of CSF leakage, the mean intraoperative loss of CSF was $118.4 \pm 56.9 \mathrm{ml}$ in the $S A H$ group and $76.3 \pm 23.0 \mathrm{ml}$ in control group. Statistical analysis found that intraoperative CSF leakage is a risk factor for postoperative SAH, which is statistically significant. It is suggested that intracranial hypotension caused by massive loss of CSF may induce venous avulsion resulting in SAH.

In addition to low intracranial pressure caused by massive intraoperative CSF leakage, complications such as acute posterior fossa syndrome, infection, $\mathrm{SAH}$, headache after dural puncture, subdural hematoma and other complications have been reported after continuous CSF drainage in the subarachnoid space ${ }^{9-12}$.For patients with CSF leakage during spinal surgery, continuous large flow of 
postoperative CSF drainage may be associated with postoperative $\mathrm{SAH}^{13}$. Sasani reported a case of $\mathrm{CBH}$ that occurred not immediately after spinal arteriovenous malformation but after lumbar and abdominal shunt ${ }^{14}$.In the same year, Miglis described a patient whose nervous system was intact after cervical discectomy and fusion, but who developed acute headache, vomiting, and visual impairment 15 hours after insertion of a lumbar drainage tube ${ }^{15}$.These reports suggest that the placement of the drainage tube may be an important cause of intracranial hemorrhage after spinal surgery by CSF shunt. This trend is also reflected in our cases, in which all patients with CSF leakage were placed with a subfascial drainage tube prior to closing the incision. In our study, we compared the mean postoperative continuous drainage volume of CSF between the two groups, and found that the continuous drainage volume per hour was $15.4 \pm 5.8 \mathrm{~mL}$ in the SAH group and $9.7 \pm 2.1 \mathrm{~mL}$ in control group. Statistical analysis showed that continuous flow of CSF was associated with SAH. Continuous low intracranial pressure due to negative pressure drainage tube placement and continuous suction has also been suggested as a possible mechanism for SAH after spinal surgery.

The deterioration of neurological symptoms after surgery may be attributed to intracranial hypotension due to persistent CSF leakage. The most typical symptoms of intracranial hypotension are headache and cerebellar dysfunction. Headache occurred in $37 \%$ of patients with lumbar puncture, which usually occurred 24 to 48 hours after dural puncture, aggravated while standing and relieved in supine position ${ }^{16}$. The headache is most likely caused by movement of the caudal side of the brain due to loss of CSF, which causes tension in the pain-sensitive dural sinuses. This type of headache occurs when there is an excess loss of CSF (about $10 \mathrm{~mL} / \mathrm{h}$ ) and $80 \%$ resolves within 5 days. However, headache is also the most common symptom of postoperative SAH and can be easily confused with intracranial hypotension. The same lethargy, altered consciousness, and dysarrhythmia can easily be incorrectly attributed to opioid overdoses. Since head CT scan after spinal surgery is not a routine examination, SAH secondary to spinal surgery may be ignored ${ }^{17}$. Therefore, we recommend that patients with unexplained severe headache, drowsiness, and altered consciousness should undergo a CT scan immediately after surgery. Patients with mild to moderate headache or confusion should be closely monitored postoperatively, and neurological examinations should be performed more frequently to prevent the disease from worsening.

The treatment of SAH after spinal surgery depends on the site and amount of bleeding and the patient's clinical status. Patients with minor bleeding without significant compression and patients without neurologic status can be managed conservatively. Patients who have massive bleeding and rapid progression of the disease must be surgically removed with or without a ventriculostomy to manage hydrocephalus. Pulled out or intermittent clamping of the drainage tube may be a safer choice to control the drainage flow. Olson et al. found in their study that intermittent drainage compared with continuous drainage can reduce the frequency and severity of cerebral vasospasm in $\mathrm{SAH}^{18}$, but continuous and large amount of CSF leakage may need to be returned to the operating room for dural repair.

\section{Conclusion:}


SAH is a very rare postoperative spinal complication. In this study, it was found that the rapid leakage of CSF caused by the rupture of the dural sac during operation and the fast flow rate of postoperative drainage fluid were closely related to the occurrence of such complications. Patients with hypertension have a higher probability of SAH. SAH is a potentially fatal complication after spinal surgery. For patients with severe headache of unknown cause after surgery, as well as patients with acute mental disorder and drowsiness not related to recent medical or laboratory abnormalities, CT scan should be performed immediately. Early detection and intervention are critical for prognosis.

\section{Abbreviations}

\begin{tabular}{|ll|}
\hline Subarachnoid hemorrhage & SAH \\
\hline cerebrospinal fluid & CSF \\
\hline
\end{tabular}

\section{Declarations}

Ethics approval and consent to participate:

Yes

\section{Consent for publication:}

Yes

\section{Availability of data and materials:}

Yes

\section{Competing interests:}

Not applicable

\section{Funding:}

Not applicable

\section{Authors' contributions}

Conceptualization: XH Zhou, J Ma 
Data curation: JQ Zhao, H Jiang

Formal analysis: JQ Zhao, J Ma

Methodology: YC Meng, C Wang

Project administration: XH Zhou

Visualization: JQ Zhao, YC Meng

Writing - original draft: JQ Zhao, H Jiang

Writing - review \& editing: XH Zhou, R Gao

\section{Acknowledgements:}

Not applicable

\section{References}

1. Van Gijn J, Kerr RS, Rinkel GJE. Subarachnoid haemorrhage[J]. The Lancet. 2007;369(9558):306-18.

2. Kaloostian PE, Kim JE, Bydon A, Sciubba DM, Wolinsky JP, Gokaslan ZL, et al. Intracranial hemorrhage after spine surgery. J Neurosurg Spine. 2013;19:370-80.

3. StorrowA B,WrennK.Aneurysmal subarachnoid hemorrhage.[J].New England Journal of Medicine,2017,354(16):1755-7.

4. Sabatino G, Della Pepa GM, Scerrati A, et al. Anatomical variants of the basal vein of Rosenthal: prevalence in idiopathic subarachnoid hemorrhage. Acta Neurochir (Wien). 2014;156:45-51.

5. Bozkurt G, Yaman ME. Subarachnoid Hemorrhage Presenting with Seizure due to Cerebrospinal Fluid Leakage after Spinal Surgery[J]. J Korean Neurosurg Soc. 2016;59(1):62-4.

6. Canh $\sim$ ao P, Falc ao F, Pinho E, et al. Vascular risk factors for perimesencephalic nonaneurysmal subarachnoid hemorrhage. J Neurol. 1999;246:492-6.

7. Sciubba DM, Kretzer RM, Wang PP. Acute intracranial subdural hematoma following a lumbar CSF leak caused by spine surgery. Spine. 2005;30:E730-2.

8. Schievink WI. Spontaneous spinal cerebrospinal fluid leaks and intracranial hypotension. JAMA. 2006;295:2286-96.

9. Chumas PD, Armstrong DC, Drake JM, et al. Tonsillar herniation: the rule rather than the exception after lumboperitoneal shunting in the pediatric population[J]. J Neurosurg. 1993;78(4):568-73.

10. Manley GT, Dillon W. Acute posterior fossa syndrome following lumbar drainage for treatment of suboccipital pseudomeningocele[J]. J Neurosurg. 2000;92(3):469-74. 
11. Roland PS, Marple BF, Meyerhoff WL, et al. Complications of lumbar spinal fluid drainage[J]. Otolaryngology-head neck surgery: official journal of American Academy of Otolaryngology-Head Neck Surgery. 1992;107(4):564.

12. Burkhard PR, Duff JM. Bilateral subdural hematomas following routine lumbar diskectomy. Headache. 2000;40:480-2.

13. Kasuya $\mathrm{H}$, Shimizu T, Kagawa $M$. The effect of continuous drainage of cerebrospinal fluid in patients with subarachnoid hemorrhage: a retrospective analysis of 108 patients.[J]. Neurosurgery. 1991;28(1):56.

14. Sasani M, Sasani H, Ozer AF. Bilateral Late Remote Cerebellar Hemorrhage as a Complication of a Lumbo-Peritoneal Shunt Applied After Spinal Arteriovenous Malformation Surgery[J]. The Journal of Spinal Cord Medicine. 2010;33(1):77-9.

15. Miglis MG, Levine DN. Intracranial venous thrombosis after placement of a lumbar drain. Neurocrit Care. 2010;12:83-7.

16. Thomas G, Jayaram H, Cudlip S, Powell M: Supratentorial and infratentorial intraparenchymal hemorrhage secondary to intracranial CSF hypotension following spinal surgery. Spine (Phila Pa 1976) 27 : E410- E412, 2002.

17. Cevik B, Kirbas I, Cakir B, Akin K, Teksam M. Remote cerebellar hemorrhage after lumbar spinal surgery. Eur J Radiol. 2009;70:7-9.

18. Olson DWM, Zomorodi M, Britz GW, et al. Continuous cerebral spinal fluid drainage associated with complications in patients admitted with subarachnoid hemorrhage[J]. J Neurosurg. 2013;119(4):974-80. 